\title{
Laryngeal Chondrosarcoma
}

\author{
Evelyn M. Potochny $\cdot$ Aaron R. Huber
}

Received: 30 June 2013/Accepted: 26 July 2013/Published online: 9 August 2013

(C) Springer Science+Business Media New York (outside the USA) 2013

\begin{abstract}
Laryngeal chondrosarcoma is a rare tumor requiring clinical, histologic, and radiographic correlation for definitive diagnosis. Although it typically presents with low-grade histology, even high-grade histology has a fairly indolent progression in this location, with a relatively low-likelihood for metastatic potential or recurrence. Because of this, conservative surgical excision with negative margins is recommended. We present a case of a laryngeal chondrosarcoma arising from the cricoid cartilage in a patient who presented with hoarseness, dysphagia, and odynophagia with subsequent head and neck computed tomography scan suggestive of the diagnosis. The patient was treated with hemicricoidectomy.
\end{abstract}

Keywords Cartilage - Chondrosarcoma - Cricoid .

Laryngeal $\cdot$ Low-grade

\section{History}

A 61-year old male with history of reflux disease status post proton-pump inhibitor treatment presented with worsening odynophagia, dysphagia, and hoarseness over a 6-month period.

\section{Radiographic Features}

Computed tomography (CT) of the head and neck showed a subglottic, mixed density mass (Fig. 1).

E. M. Potochny $(\bowtie) \cdot$ A. R. Huber

Naval Medical Center San Diego, San Diego, CA, USA

e-mail: evelyn.potochny@med.navy.mil

\section{Diagnosis}

Gross pathology from a biopsy of the cricoid mass consisted of numerous fragments of soft tissue, with roughened external surfaces and smooth, lobular, semi-translucent cut surfaces. Histological examination of the biopsy showed hyaline cartilage comprised of lobules of multinucleated chondrocytes with increased nucleus:cytoplasmic (N:C) ratios (Figs. 2, 3). Mitotic activity was not identified. There was no evidence of a myxoid matrix in any of the fields, nor were there any areas of ischemia, infarction, or necrosis. An area of benign cartilage consistent with a chondroma was appreciated (Fig. 4). These findings are diagnostic of a low-grade (grade 1 of 3) chondrosarcoma of the larynx.

\section{Discussion}

Laryngeal chondrosarcoma of the larynx is a rare tumor, comprising less than $1 \%$ of sarcomas [1]. The tumor most commonly arises from the cricoid, and less frequently from the arytenoid and thyroid cartilage or the epiglottis itself $[1,2]$. The most common symptoms include dyspnea, hoarseness, dysphagia, odynophagia, and airway obstruction $[1,3]$. Men are more commonly affected than women and the age of presentation is usually in the 6th decade, with a range between 50 and 70 years [1]. It has been found to be associated with hyaline cartilage primarily, and with elastic cartilage only rarely [1].

Imaging, including radiograph, CT or magnetic resonance imaging (MRI), is of utmost importance in suggesting a chondroid neoplasm. A variably dense, expansile lesion with characteristic "popcorn" calcifications is usually seen $[1,4,7]$. Invasion into adjacent soft tissue or bone 


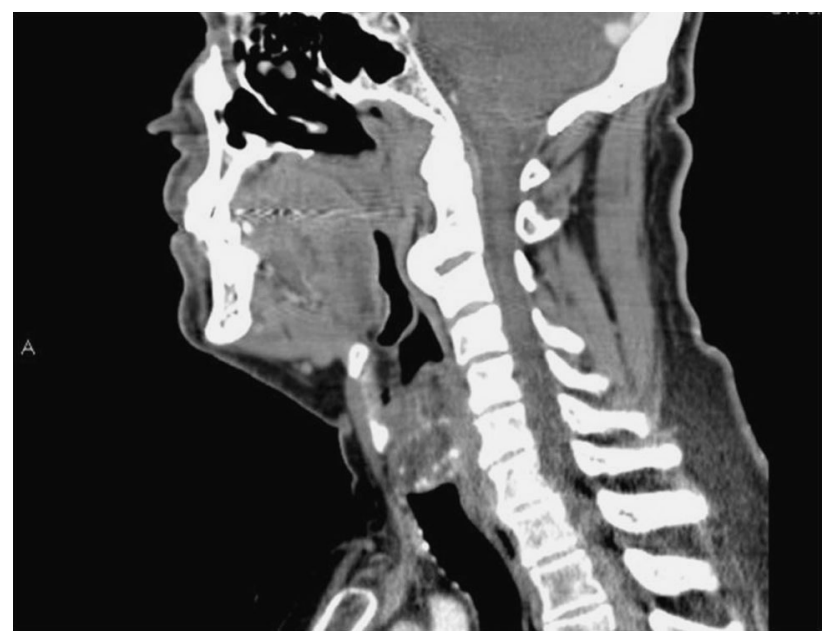

Fig. 1 Computed tomography of the head and neck showed a subglottic, mixed density mass

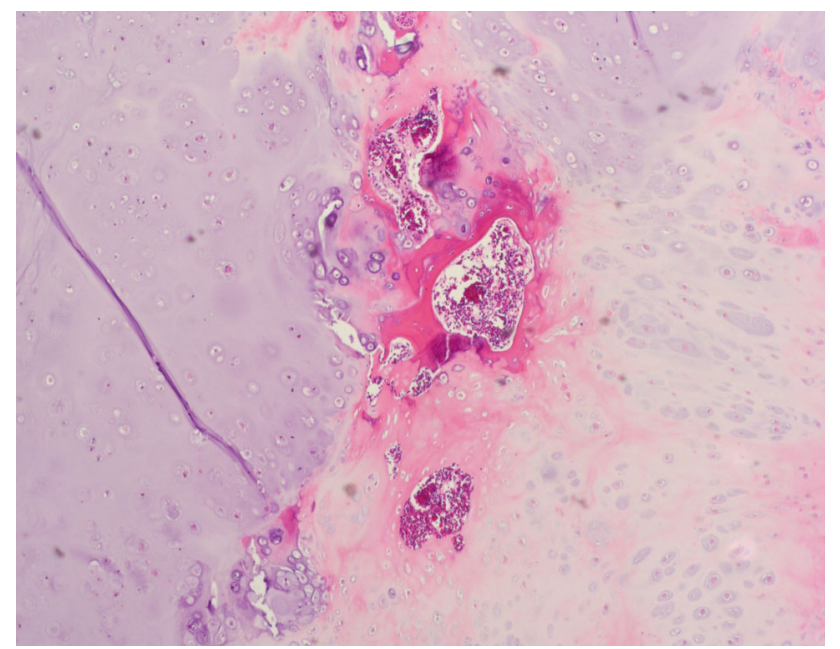

Fig. 2 Low-grade chondrosarcoma (left) abutting benign hyaline cartilage (right)

may be present $[1,3,4]$. Imaging cannot definitively differentiate a benign chondroma from a chondrosarcoma, since the majority of chondrosarcomas of the larynx are low-grade histologically and $62 \%$ of chondrosarcomas have an associated chondroma [1].

Gross findings consistently show smooth, lobular, translucent tissue portions. The specimen is often received fragmented. Tumors range in size from less than $1 \mathrm{~cm}$ to up to $10 \mathrm{~cm}$, but size has never been shown to correlate with prognosis [1].

Histological findings of chondrosarcomas demonstrate hyaline cartilage of variable degrees of cellularity, anaplasia, and mitotic index. Cellularity, mitotic activity, and nuclear size are key features in grading and distinguishing benign from malignant tumors $[1,3-6]$. Since the majority of lesions are low-grade, the distinction from benign

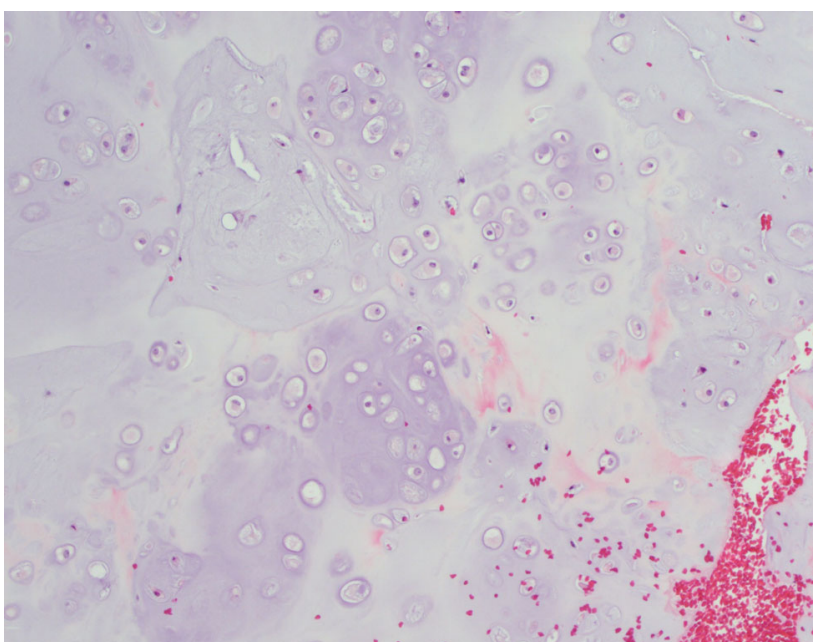

Fig. 3 Mildly cellular field with predominantly small mononucleated, atypical chondrocytes with ample cytoplasm, consistent with low-grade (grade 1) cytomorphology

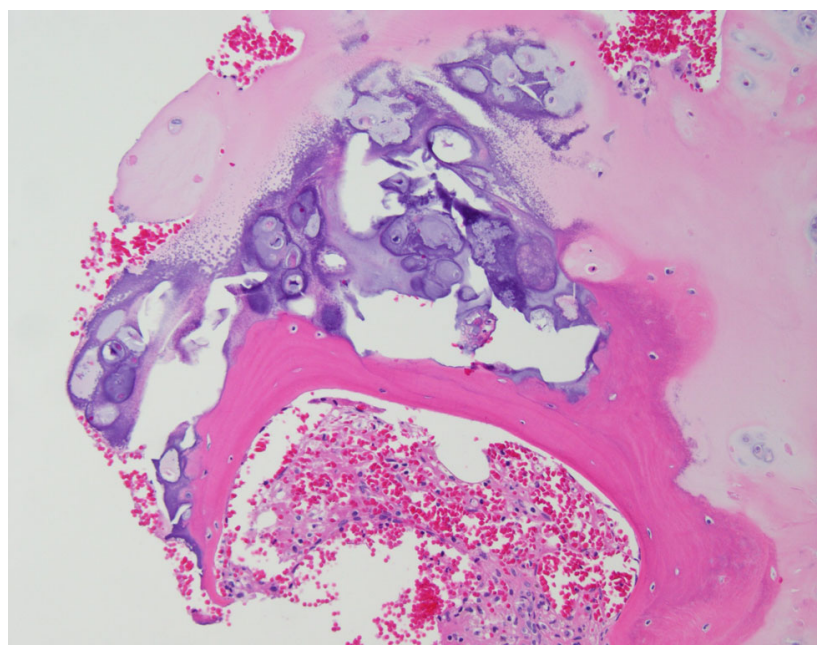

Fig. 4 Focus of degenerating (ischemic) benign chondroma

chondromas must be made. Chondromas are overall hypocellular, with predominantly small, mononucleated chondrocytes that retain normal $\mathrm{N}: \mathrm{C}$ ratios and show no hyperchromasia, mitotic activity, or necrosis and may reside in an eosinophilic background matrix [1, 3-5, 7]. Grade 1 chondrosarcomas demonstrate bi- or multinucleated chondrocytes with areas of calcification but which still lack mitotic activity. Grade 2 chondrosarcomas show increased cellularity with rare mitotic forms, but which still demonstrate relatively low N:C ratios. High-grade (grade 3) chondrosarcomas, on the other hand, are hypercellular with chondrocytes which are consistently multinucleated, with increased mitotic activity. A myxoid matrix has been associated with more aggressive behavior [1]. Chondrosarcomas may be juxtaposed next to benign chondromas, and as Thompson and Gannon have noted, may show 
unique ischemic changes: linear basophilic areas or granular calcifications in areas where the benign component merges with the malignant component $[1,4,5]$.

Metastatic potential is rare in grade I chondrosarcomas, and seen in only $10 \%$ of grade 2 lesions [6]. While there has been documentation of $71 \%$ of high-grade laryngeal chondroasarcomas showing metastases, a study of 111 cases conducted through the Armed Forces Institute of Pathology found only $1.9 \%$ of cases developed metastases $[1,6]$. Recurrence rates cited in the literature have ranged from 18 to $40 \%[1,4]$. The overall survival, regardless of grade, has been cited as high as $95 \%$ at 10 years [1]. Because of this, conservative therapy is the generally treatment of choice [1, 5, 7]. Total laryngectomy is reserved for large tumors in which surgery would cause destabilization of the cricoid ring [7].

\section{References}

1. Thompson LDR, Gannon FH. Chondrosarcoma of the larynx: a clinicopathologic study of 111 cases with a review of the literature. Am J Surg Pathol. 2002;26(7):836-51.

2. Kozelsky TF, Bonner JA, Foote RL, et al. Laryngeal chondrosarcomas: the mayo clinic experience. J Surg Oncol. 1997;65:269-73.

3. Thompson LDR. Chondrosarcoma of the larynx. ENT J. 2004; 83(9):609.

4. Buda I, Hod R, et al. Chondrosarcoma of the larynx. Isr Med Assoc J. 2012;14(11):681-4.

5. Lichtenstein L, Jaffe HL. Chondrosarcoma of bone. Am J Pathol. 1943;19:553-74.

6. Evans HL, Ayala AG, Romsdahi MM. Prognostic factors in chondrosarcoma of bone. Cancer. 1977;40:818-31.

7. Casiraghi O, Martinez-Madrigal F, et al. Chondroid tumors of the larynx: a clinicopathologic study of 19 cases, including two dedifferentiated chondrosarcomas. Ann Diagn Pathol. 2004;8:189-97. 